\title{
Repurposing Methotrexate in Dampening SARS-CoV2-S1-Mediated IL6 Expression: Lessons Learnt from Lung Cancer
}

\author{
Pruthvi Gowda $^{1}$, Shruti Patrick ${ }^{1}$, Shanker Datt Joshi ${ }^{1}$, Rajesh Kumar Kumawat ${ }^{1}$ and \\ Ellora $\operatorname{Sen}^{1,2}$ (i)
}

\begin{abstract}
Received 3 May 2021; accepted 5 August 2021
Abstract_L Severe acute respiratory syndrome coronavirus 2 (SARS-CoV-2) infection (COVID-19) is associated with uncontrolled inflammatory responses. Loss of pulmonary angiotensin-converting enzyme 2 (ACE2) function has been associated with SARS$\mathrm{CoV}-2$ infection. The aberrant signalling and dysregulated inflammation characteristic of lung cancer have marked similarities with SARS-CoV-2 infection. Spearman's correlation analysis of The Cancer Genome Atlas (TCGA) datasets indicated an inverse correlation between $A C E 2$ and $I L 6$ in lung adenocarcinoma. qRT-PCR analysis revealed CoV2-SRBD-mediated diminished $A C E 2$ expression in lung cancer cells that was concomitant with increased $I L 6$ expression. Western blot and qRT-PCR analysis suggested that treatment with methotrexate (MTx) dampened CoV-2-SRBD-mediated increase in JAK1/STAT3 phosphorylation, gp130, IL6, and folate-binding protein (FBP) expressions. MTx also rescued the diminished expression of ACE2 in CoV-2-SRBD transfected cells. As lung tissue injury in severely affected COVID-19 patients is characterised by aberrant inflammatory response, repurposing MTx as an effective therapy against critical regulators of inflammation in SARS-CoV-2 infection warrants investigation.
\end{abstract}

KEY WORDS: COVID-19; ACE2; IL-6; Folate-binding protein; Methotrexate.

\section{INTRODUCTION}

The binding of SARS-CoV-2-S (spike) protein to human angiotensin-converting enzyme 2 (hACE2) is a crucial step in cell entry mechanism of SARS-CoV-2 [1]. The receptor-binding domain (RBD) in the $\mathrm{S} 1$ subunit

\footnotetext{
${ }^{1}$ National Brain Research Centre, Manesar, Gurgaon, Haryana 122 052, India

${ }^{2}$ To whom correspondence should be addressed at National Brain Research Centre, Manesar, Gurgaon, Haryana 122 052, India. Email: ellora@nbrc.ac.in
}

of SARS-CoV-2 is important for binding to the ACE2 receptor protein. SARS-CoV spike protein-mediated ACE2 downregulation has been suggested to contribute to the severity of lung pathologies [2]. SARS-CoV-2 infection is associated with uncontrolled inflammatory responses [3]. Given the tissue-protective component of ACE2 during severe acute lung injury [2], understanding its correlation with pro-inflammatory mediators in an inflammatory setting could provide insight into events associated with the hyper-inflammation that characterizes COVID-19. 
A
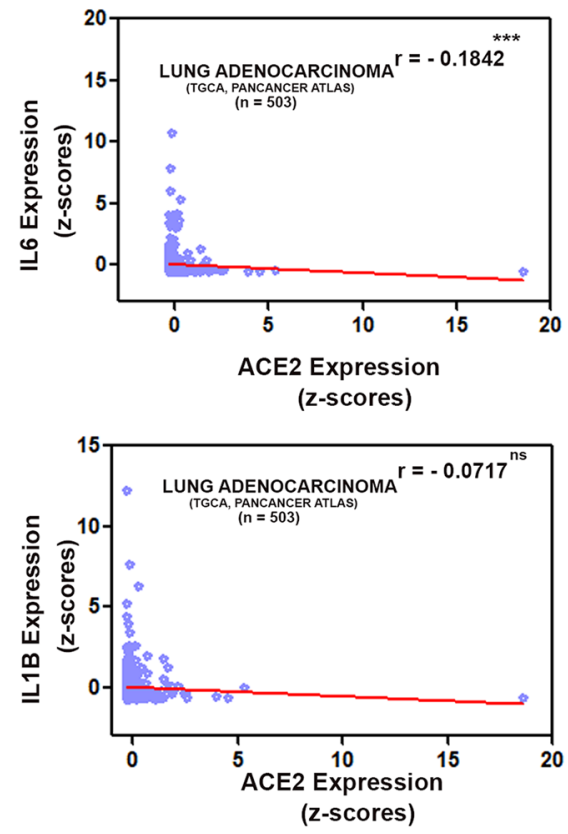

B

A549 (Lung)

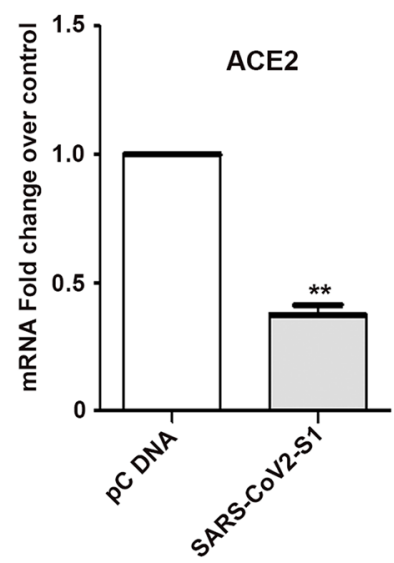

C A549 (Lung)

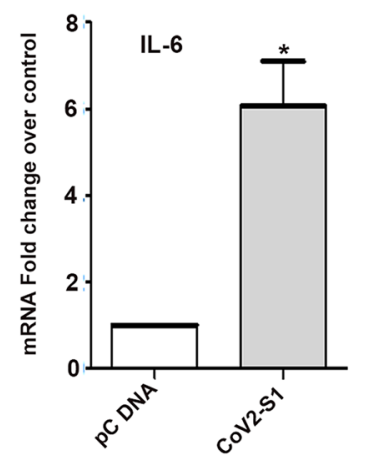

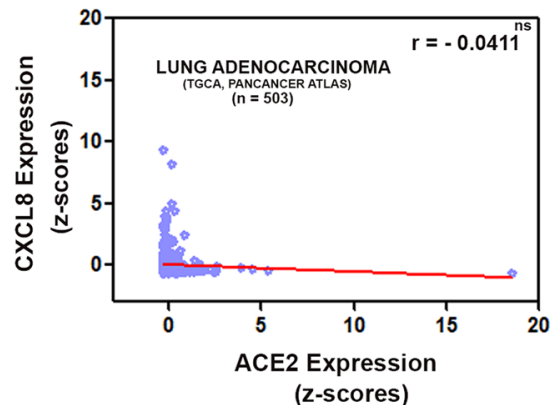

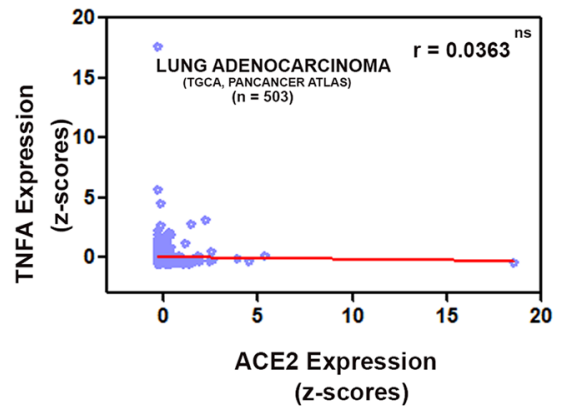

H1299 (Lung)
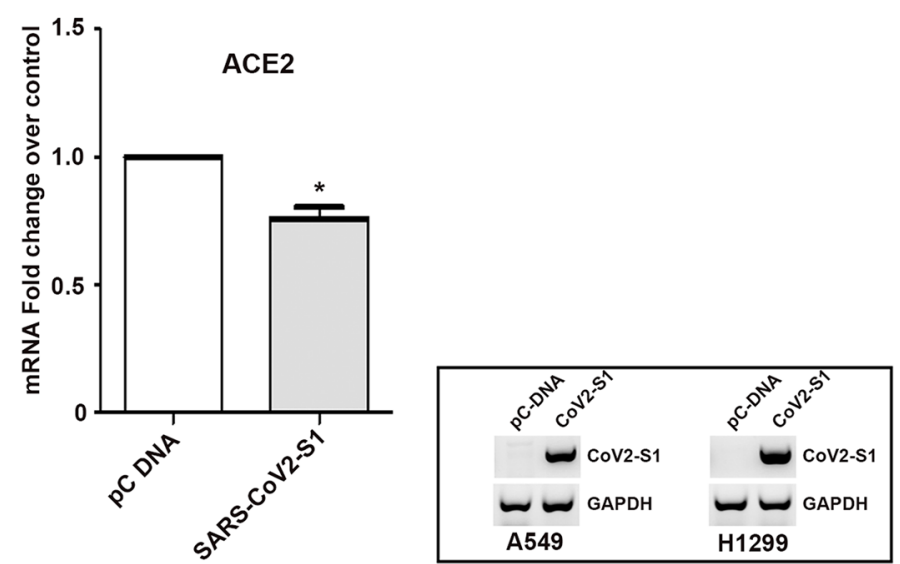

H1299 (Lung)

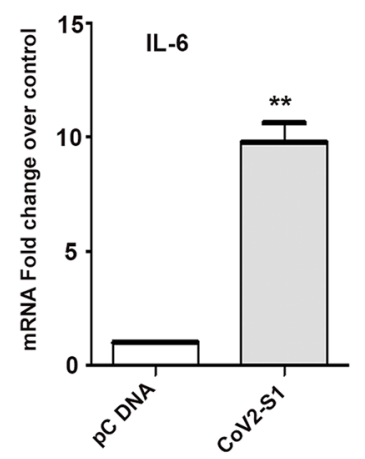


4Fig. 1 ACE2 is negatively correlated with IL-6. Correlation analyses of TCGA datasets indicate a negative correlation between IL6 and $A C E 2$ in lung cancer. $r$ Values were generated by a linear-fit model using GraphPad Prism. Individual correlation of $A C E 2$ with $C X C L 8$, $I L 1 B, T N F A$, and IL6. IL6 was found to be significantly correlated with $A C E 2$ expression. ${ }^{*} P<0.05$; ${ }^{*} * P<0.01$; $* * * P<0.001, r$, Spearman's rank correlation coefficient. B Real-time PCR indicating the expression levels of ACE2 and C IL6 in two lung cancer cells (A549, H1229) transfected with SARS-CoV2-SRBD. Inset shows transfection efficiency of SARS-CoV2-SRBD in the cell lines. Lung cells transfected with empty vector are used as a control for SARS-CoV2-SRBD transfected lung cells.

Lung cancer growth and metastases are accompanied by dramatic decrease in both lung and serum ACE activity, with conformational fingerprinting demonstrating significantly altered ACE expression and activity in tumour lung tissues [4]. Interestingly, the use of ACE inhibitors is associated with an increased risk of lung cancer [5]. As ACE2 expression is associated with tumorigenesis in lung cancer [6], and considering the common involvement of ACE2 in lung cancer and SARS-CoV-2 infection, we analysed the patient lung cancer dataset from The Cancer Genome Atlas (TCGA) to find the correlations between ACE2 and genes associated with pro-inflammatory responses. We surmised that viewing SARS-CoV-2 through the lens of lung cancer would provide understanding of the host responses in COVID19 infection through insights gained from lung cancer. Moreover, comparison of the two diseases showing commonalities might indicate ways in which therapies developed for one disease might be applied to the other, as has been suggested previously [7]. A better understanding of the convergences between critical players in the ACE2inflammatory network would enable efficient repurposing of approved lung cancer therapeutics for COVID-19 infection.

\section{MATERIALS AND METHODS}

\section{Analysis of Lung Cancer Data}

For studying the genes co-expressed with ACE2 in lung cancer, transcriptome sequencing (RNA-seq) data from TCGA lung adenocarcinoma dataset was downloaded from cBioPortal [8]. Spearman's rank correlation coefficient for RNA expression values of IL6, and ACE2 in lung cancer, was calculated using GraphPad PrismV5 statistical software.

\section{Cell Culture}

Human lung cell carcinoma line A549 (CCL-185, ATCC) was grown in Dulbecco's modified eagle medium: nutrient mixture F-12 (DMEM/F-12) (\#12,400-024, Gibco). Human lung cancer cell line NCI-H1299 (CRL5803, ATCC) was grown in RPMI-1640 medium (R6504, Sigma-Aldrich). All culture media were supplemented with $10 \%$ heat-inactivated foetal bovine serum (HI-FBS) (\#16,140, Gibco) and penicillin (100 U/mL)-streptomycin (100 $\mu \mathrm{g} / \mathrm{mL})(\# 15,140$, Gibco).

\section{Treatment and Transfection}

Transfections were done as described previously [9]. Briefly, cells were grown in their respective culture media and upon reaching $70 \%$ confluence the media was replaced with OptiMEM $(31,985$; Gibco) $2 \mathrm{~h}$ prior to transfection. The cells were transfected with pcDNA SARS-CoV-2-S-RBD or the corresponding empty vector using Lipofectamine 2000 transfection reagent (\#11,668,027; Invitrogen). Transfected cells were either harvested for further processing or serum starved for $6 \mathrm{~h}$, after which they were treated with $0.5 \mu \mathrm{M}$ methotrexate (MTx) (M9929; Sigma) for $24 \mathrm{~h}$.

\section{Western Blot Analysis}

Proteins isolated from cells transfected with pcDNA SARS-CoV-2-S-RBD in the presence and absence of MTx were electrophoresed on polyacrylamide gels, and Western blot was performed as described previously [9], using antibodies against phospho-JAK1 (3331; Cell Signalling Technologies), JAK1 (3332; Cell Signalling Technologies), PhosphoSTAT3 (9145; Cell Signalling Technologies), STAT3 (9132; Cell Signalling Technologies), FBP (ab3361; Abcam), and gp130 (sc656; Santa Cruz Biotechnology). Secondary antibodies were purchased from Vector Laboratories Inc. After the addition of enhanced chemiluminescent reagent (Millipore), blots were exposed to Chemigenius Bioimaging System (Syngene) for development, and images were captured using GeneSnap software (Syngene). The blots were stripped and re-probed with anti- $\beta$-actin (A3854; Sigma-Aldrich) to determine equivalent loading as described previously [9]. 
A

A549

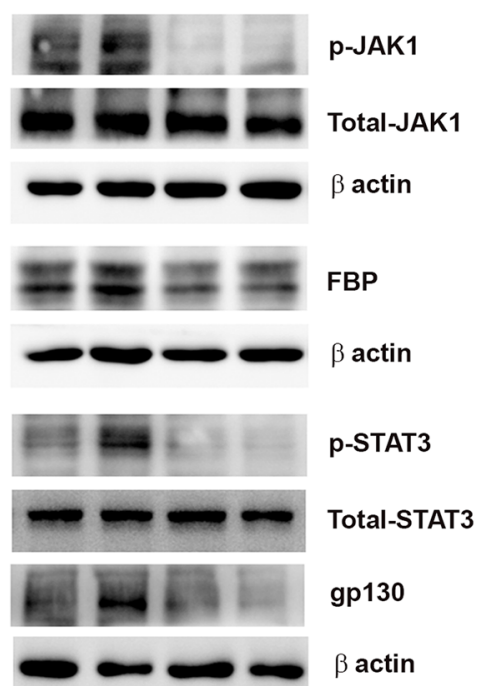

$\begin{array}{rllll}\text { PC DNA } & + & - & + & - \\ \text { CoV2-S1 } & - & + & - & + \\ \text { MTX } & - & - & + & +\end{array}$

C

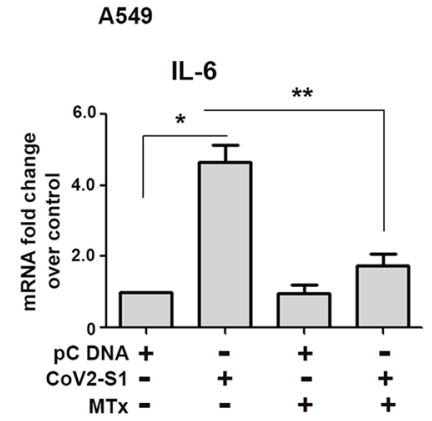

H1299

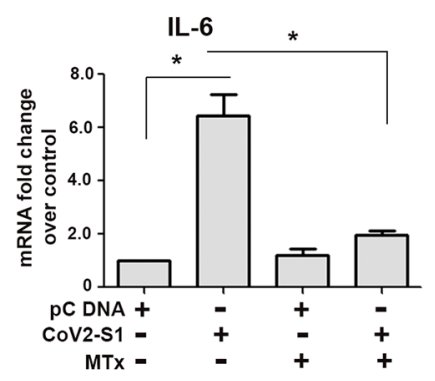

B

\section{H1299}

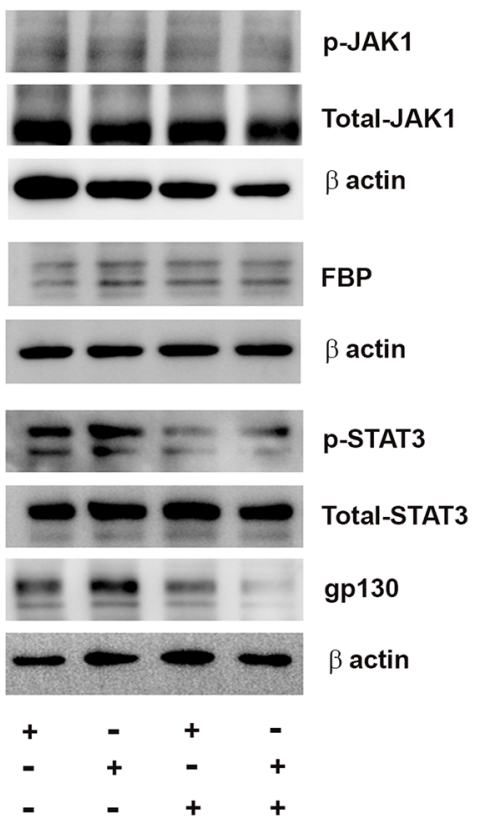

E

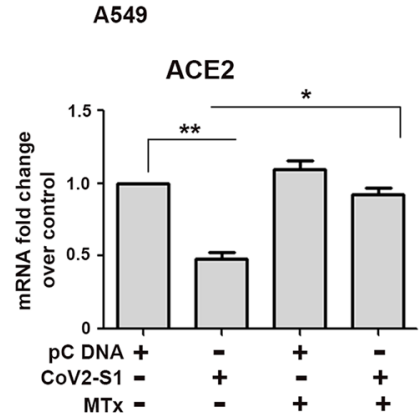

H1299

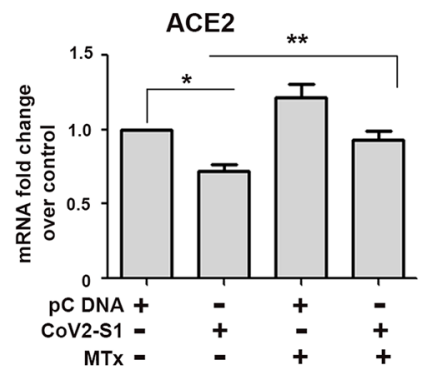

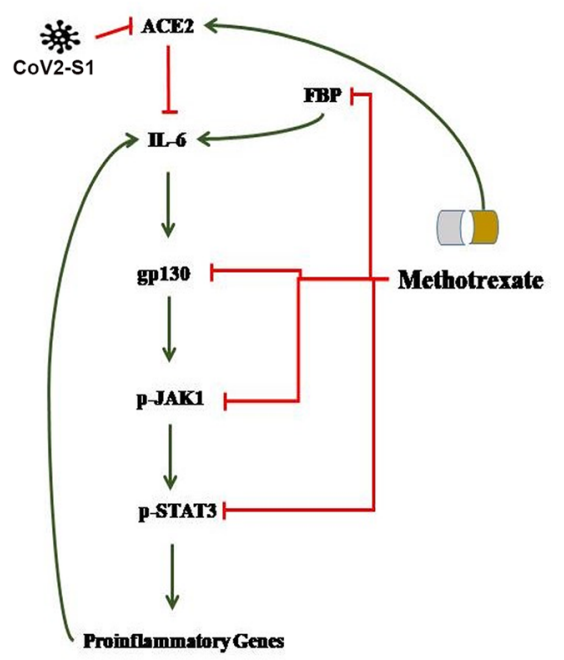


Fig. 2 A, B The phosphorylation of JAK (Tyr1034/1035) and STAT3 (Tyr705) in lung cells transfected with SARS-CoV-2 SRBD in the presence and absence of methotrexate were analysed by Western blotting. The effect of MTX on the expression of gp130 and FBP in SARS-CoV-2 S-RBD transfected cells is shown. Levels of total JAK and STAT3 are also shown. Blots are representative of data from two independent experiments with similar results. Blots were stripped and re-probed with $\beta$-actin to establish equivalent loading. C qRT-PCR analysis shows that MTX abrogates SARS-CoV-2 S-RBD induced an increase in IL-6 expression, and $\mathbf{D}$ rescues diminished ACE2 expression in transfected lung cells. The graphs represent average fold change values from three independent experiments. E Schematic representing the regulation of ACE2 and IL- 6 regulators by methotrexate. The graphs represent average fold-change values from three independent experiments. $p<0.05^{*}, p<0.01 * *$.

\section{Quantitative Real-Time PCR}

To analyse ACE2 and IL6 mRNA levels in lung cells overexpressing pcDNA SARS-CoV-2-S-RBD in the presence or absence of MTx, RNA was isolated using RNeasy kit (Qiagen) and cDNA was synthesised using High-Capacity cDNA Reverse Transcription Kit (Applied Biosystems) on Veriti Thermal cycler (Applied Biosystems). Real-time PCR was performed as described [9] using Quant Studio V (Applied Biosystems Inc.). All samples were normalised with their respective 18S rRNA Ct values, and results were plotted as fold change over control for each mRNA transcript. The qPCR primers used are as follows:

\begin{tabular}{lcc}
\hline S. No & Gene & Primer sequences \\
\hline 1 & IL6 & Forward: GCCTTCGGT \\
& & CCAGTTGCCTT \\
& & Reverse: AGTGCCTCTTTG \\
& CTGCTTTCA \\
2 & & Forward: GGACCCAGG \\
& ACE2 & AAATGTTCAGA \\
& & Reverse: GGCTGCAGA \\
& & AAGTGACATGA \\
& & Forward: TTCGGTGAG \\
& CoV2- & GTTTTAACGC \\
& SRBD & Reverse: TTTGTCGGA \\
& TCCCTTTTGG \\
\hline
\end{tabular}

\section{Statistical Analysis}

All correlation tests were performed using Spearman's rank correlation analysis. Comparison between groups was done using paired two-tailed Student's $t$ test.
All $p$-values less than 0.05 were taken as significant. Statistical analysis was performed using GraphPad PrismV5.

\section{RESULTS}

\section{Correlation Between Pro-Inflammatory Mediators and ACE2 in Lung Cancers}

As ACE2 expression is associated with tumorigenesis in lung cancer [6], analysis of the TCGA dataset was performed to investigate the association between ACE2 and pro-inflammatory genes in lung cancer patients. A negative correlation between pro-inflammatory IL6 and ACE2 was noted (Fig. 1a). However, no significant associations between ACE2 and other cytokines such as IL1B, TNFA, and CXCL8 were found (Fig. 1a).

\section{CoV-2-S1-Mediated Diminished ACE2 Expression Is Accompanied with Enhanced Expression of IL6}

The binding of SARS-CoV-2-SRBD to ACE2 is crucial in cell entry mechanism of SARS-CoV-2 [1]. A decrease in ACE2 expression was observed in lung cells (A549 and H1299) transfected with SARS-CoV2-S-RBD as compared to cells transfected with empty vector (Fig. 1b). Inset shows the efficacy of SARS-CoV2-S-RBD transfection. As TCGA data reanalysis indicated inverse correlation between IL6 and ACE2; the status of IL6 in CoV2-SRBD transfected lung cells was investigated. Decreased ACE2 expressions in CoV2SRBD transfected lung cells (A549 and H1299) were concomitant with increase in IL6 (Fig. 1c).

\section{Methotrexate Diminishes CoV2-S1-Mediated Increase in JAK/STAT Phosphorylation, IL6, and ACE2 Expressions}

ACE2 is known to mediate the upstream regulators of JAK/STAT pathway [10], and IL-6/JAK1/ STAT3 signalling pathway is aberrantly hyperactivated in chronic inflammatory conditions [11]. Consistent with elevated IL6 expression, elevated phosphorylation of JAK1 and STAT3 was observed in CoV2SRBD transfected lung cells (Fig. 2a, b). MTx, a folic acid analogue, inhibits JAK/STAT pathway [12] and suppresses IL6 gene expression [13]. Increase in IL6 
levels in CoV2-S1 transfected lung cells prompted us to examine the effect of MTx on JAK1/STAT3 phosphorylation. Treatment with MTx significantly attenuated CoV2-S1-mediated increase in JAK1 and STAT3 phosphorylation (Fig. 2a, b) as well as increase in IL6 expression (Fig. 2c). Given the negative correlation between ACE2 and IL6, MTx-mediated decrease in IL6 expression (Fig. 2c) was accompanied by increase in ACE2 expression (Fig. 2d).

\section{Methotrexate Diminishes gp130 and FBP Levels in CoV2-S1 Transfected Cells}

MTx inhibits dehydrofolate reductase (DHFR) - $\mathrm{a}$ critical folate cycle enzyme. FR $\alpha$ or FBP, a folate-binding protein is one of several methods by which folate, is taken up by cells. Folic acid binding to the FBP mediates STAT3 activation and enhances the JAK/STAT signalling pathway [14]. Besides, gp130 which serves as a transducing receptor for folic acid-induced FBP-STAT3 signal [14] is upstream of STAT3/JAK signalling pathway [15]. On investigating the status of FBP and gp130 in CoV2-S1 transfected lung cells exhibiting heightened JAK1/STAT3 activation, an increase in both was observed (Fig. 2a, b). SRBD-mediated increase in both FBP and gp130 was abrogated in the presence of MTx (Fig. 2a, b).

\section{DISCUSSION}

Our finding has highlighted that intracellular signalling networks in lung cancer can be extrapolated to explain the aberrant inflammatory response in COVID-19 infection. The negative correlation of ACE2 with IL-6 levels, as predicted from TCGA lung cancer datasets, was also noted in lung cells transfected with SARS-CoV-2SRBD. Downregulation of ACE2 was concomitant with elevated IL6 expression in SARS-CoV-2-SRBD transfected lung cells. IL6 not only facilitates development and progression of K-Ras mutant lung cancer [16] but also serves as a prognosticator in COVID-19 patients [17]. SARS-CoV2 S-RBD-mediated increase in IL-6 is reported in lung cells [18]. Clinical trial with IL-6R antagonist sarilumab has indicated the benefits of early intervention with IL6 modulatory therapies for COVID19 [19]. Based on these findings, several large-scale trials evaluating the efficacy of IL6 modulatory therapies have been initiated. One such clinical trial with tocilizumab (a recombinant humanized anti-IL-6 receptor) is in patients with severe worsening COVID-19 pneumonia and high inflammatory parameters [20]. Since targeting the IL6/JAK1/ STAT3 signalling pathway inhibits tumour growth, inhibitors of this axis are currently in clinical and/or preclinical development for several malignancies including lung cancers [11]. Our finding underscores the potential of MTx to not only rescue CoV-2-SRBD-induced JAK1-STAT3IL6 pathway but also dampens gp130 and FBP expression (Fig. 2e).

Overexpression of folate receptor alpha in lung cancer has prognostic and diagnostic significance [21], and among the several FDA-approved drugs for lung cancer, folate inhibitors such as methotrexate and Pemetrexed disodium find a place. An ongoing phase II clinical trial is evaluating farletuzumab (a monoclonal antibody targeting FOLR1) in patients with lung adenocarcinoma [22]. Importantly, methotrexate prevents human cytomegalovirus (HCMV) replication [23], and FOLR1 serves as a significant cofactor for cellular entry for Marburg and Ebola viruses [24]. As the signalling pathways associated with inflammation show striking similarities between SARS-CoV-2 infection and lung cancers, repurposing of anti-lung cancer drugs for COVID-19 has considerable promise.

As the host response plays a determinant role in the pathogenesis of infectious diseases, strategies for treatment need to consider both the nature of host response as well as the microbial factors. The concept of "repurposing" or finding use of an existing anti-inflammatory marketed drug for the treatment of COVID-19 is emerging as a prominent strategy. Methotrexate has been recognized by the World Health Organization as an "essential medication" because of its dynamic range of anti-inflammatory mechanisms. Though methotrexate therapy has shown promises in fulminant inflammatory conditions and continues to be a cornerstone in the treatment of autoimmune diseases and many neoplastic disorders, oral administration of MTx takes several weeks to build up effect [25]. In patients with inadequate responsiveness to MTX alone, combinatorial therapy with IL-6 inhibitor tocilizumab has been found to be effective [26]. A recent study from our lab have indicated heightened levels of High mobility group box 1 (HMGB1) in SARS-CoV2-S-RBD transfected cells [18]. Extracellular release of proinflammatory mediator HMGB1 during infection or sterile tissue injury involves its translocation from nucleus to cytoplasm, and JAK/STAT1 is crucial for HMGB1 nuclear 
translocation [27]. Inhibition of HMGB1 by glycyrrhizin not only prevented SARS-COV2-mediated release of IL-6 in lung cells, but also prevented viral replication [18]. As inhibition of HMGB1 by glycyrrhizin suppresses activation of JAK-STAT pathway, combinatorial therapy of MTx and glycyrrhizin warrants investigation. Our study raises the possibility that this inexpensive and widely available agent could be repurposed for calming hyper-inflammation in COVID-19 infection.

Abbreviations ACE2, Angiotensin-converting enzyme 2; TCGA, The Cancer Genome Atlas; IL-1 $\beta$, Interleukin 1 beta; IL6, Interleukin 6; $\mathrm{TNF} \alpha$, Tumour necrosis factor; FOLR1, Folate receptor $\alpha$

\section{AUTHOR CONTRIBUTION}

PG and ES designed the study. PG, SP, SDJ, and RKK performed data acquisition. PG, SP, and ES wrote, read, and approved the manuscript.

\section{FUNDING}

The work was supported by NBRC core funds.

\section{AVAILABILITY OF DATA AND MATERIAL}

The datasets used and/or analysed during the current study are available from the corresponding author on reasonable request.

\section{DECLARATIONS}

Ethics Approval and Consent to Participate. Not applicable.

Consent for Publication. Not applicable.

Conflict of Interest The authors declare no competing interests.

\section{REFERENCES}

1. Shang, J., Y. Wan, C. Luo, G. Ye, Q. Geng, A. Auerbach, et al. 2020. Cell entry mechanisms of SARS-CoV-2. Proc Natl Acad Sci U S A. 117 (21): 11727-11734.

2. Kuba, K., Y. Imai, S. Rao, H. Gao, F. Guo, B. Guan, et al. 2005. A crucial role of angiotensin converting enzyme 2 (ACE2) in SARS coronavirus-induced lung injury. Nature medicine. 11 (8): 875-879.
3. Chakraborty S, Basu A. The COVID-19 pandemic: catching up with the cataclysm. F1000Res. 2020;9.

4. Danilov SM, Metzger R, Klieser E, Sotlar K, Trakht IN, Garcia JGN. Tissue ACE phenotyping in lung cancer. PLoS One. 2019;14(12):e0226553.

5. Hicks BM, Filion KB, Yin H, Sakr L, Udell JA, Azoulay L. Angiotensin converting enzyme inhibitors and risk of lung cancer: population based cohort study. BMJ. 2018;363:k4209.

6. Feng, Y., H. Wan, J. Liu, R. Zhang, Q. Ma, B. Han, et al. 2010. The angiotensin-converting enzyme 2 in tumor growth and tumor-associated angiogenesis in non-small cell lung cancer. Oncology Reports 23 (4): 941-948.

7. Seeger-Nukpezah T, Geynisman DM, Nikonova AS, Benzing T, Golemis EA. The hallmarks of cancer: relevance to the pathogenesis of polycystic kidney disease. Nat Rev Nephrol.11(9):515-34.

8. Gao J, Aksoy BA, Dogrusoz U, Dresdner G, Gross B, Sumer SO, et al. Integrative analysis of complex cancer genomics and clinical profiles using the cBioPortal. Science signaling.6(269):pl1.

9. Gowda P, Patrick S, Singh A, Sheikh T, Sen E. Mutant isocitrate dehydrogenase 1 disrupts PKM2-beta-catenin-BRG1 transcriptional network-driven CD47 expression. Mol Cell Biol.38(9).

10. Satou, R., and R.A. Gonzalez-Villalobos. 2012. JAK-STAT and the renin-angiotensin system: The role of the JAK-STAT pathway in blood pressure and intrarenal renin-angiotensin system regulation. JAKSTAT. 1 (4): 250-256.

11. Johnson, D.E., R.A. O'Keefe, and J.R. Grandis. 2018. Targeting the IL-6/JAK/STAT3 signalling axis in cancer. Nature Reviews. Clinical Oncology 15 (4): 234-248.

12. Thomas S, Fisher KH, Snowden JA, Danson SJ, Brown S, Zeidler MP. Methotrexate Is a JAK/STAT pathway inhibitor. PLoS One. 2015;10(7):e0130078.

13. Sung, J.Y., J.H. Hong, H.S. Kang, I. Choi, S.D. Lim, J.K. Lee, et al. 2000. Methotrexate suppresses the interleukin-6 induced generation of reactive oxygen species in the synoviocytes of rheumatoid arthritis. Immunopharmacology 47 (1): 35-44.

14. Hansen, M.F., E. Greibe, S. Skovbjerg, S. Rohde, A.C. Kristensen, T.R. Jensen, et al. 2015. Folic acid mediates activation of the prooncogene STAT3 via the Folate Receptor alpha. Cellular Signalling 27 (7): 1356-1368.

15. Hirano, T., K. Ishihara, and M. Hibi. 2000. Roles of STAT3 in mediating the cell growth, differentiation and survival signals relayed through the IL-6 family of cytokine receptors. Oncogene 19 (21): 2548-2556

16. Caetano, M.S., H. Zhang, A.M. Cumpian, L. Gong, N. Unver, E.J. Ostrin, et al. 2016. IL6 Blockade reprograms the lung tumor microenvironment to limit the development and progression of K-ras-mutant lung cancer. Cancer Research 76 (11): 3189-3199.

17. Grifoni, E., A. Valoriani, F. Cei, R. Lamanna, A.M.G. Gelli, B. Ciambotti, et al. 2020. Interleukin-6 as prognosticator in patients with COVID-19. Journal of Infection 81 (3): 452-482.

18. Gowda P, Patrick S, Joshi SD, Kumawat RK, Sen E. Glycyrrhizin prevents SARS-CoV-2 S1 and Orf3a induced high mobility group box 1 (HMGB1) release and inhibits viral replication. Cytokine. 2021;142:155496.

19. Montesarchio V, Parrela R, Iommelli C, Bianco A, Manzillo E, Fraganza F, et al. Outcomes and biomarker analyses among patients with COVID-19 treated with interleukin 6 (IL-6) receptor antagonist sarilumab at a single institution in Italy. J Immunother Cancer. 2020;8(2).

20. Roumier, Mathilde, Romain Paule, Alexandre Vallée, Julien Rohmer, Marie Ballester, Anne-Laure. Brun, Charles Cerf, 
Marie-Laure. Chabi, Thierry Chinet, Marie-Alice. Colombier, Eric Farfour, Erwan Fourn, Guillaume Géri, David Khau, Ibrahim Marroun, Matthieu Ponsoye, Antoine Roux, Hélène. Salvator, Yoland Schoindre, Anne-Gaëlle. Si Larbi, Colas Tchérakian, Marc Vasse, Anne Verrat, Benjamin Zuber, Louis-Jean. Couderc, Jean-Emmanuel. Kahn, Matthieu Groh, and Félix. Ackermann. 2021. Tocilizumab for Severe Worsening COVID19 Pneumonia: a Propensity Score Analysis. Journal of Clinical Immunology 41 (2): 303-314. https://doi.org/10.1007/ s10875-020-00911-6.

21. O'Shannessy, D.J., G. Yu, R. Smale, Y.S. Fu, S. Singhal, R.P. Thiel, et al. 2012. Folate receptor alpha expression in lung cancer: Diagnostic and prognostic significance. Oncotarget 3 (4): 414-425.

22. Thomas, A., J. Maltzman, and R. Hassan. 2013. Farletuzumab in lung cancer. Lung Cancer 80 (1): 15-18.

23. Lembo, D., G. Gribaudo, R. Cavallo, L. Riera, A. Angeretti, L. Hertel, et al. 1999. Human cytomegalovirus stimulates cellular dihydrofolate reductase activity in quiescent cells. Intervirology 42 (1): 30-36.

24. Chan, S.Y., C.J. Empig, F.J. Welte, R.F. Speck, A. Schmaljohn, J.F. Kreisberg, et al. 2001. Folate receptor-alpha is a cofactor for cellular entry by Marburg and Ebola viruses. Cell 106 (1): 117-126.

25. Bello, A.E., E.L. Perkins, R. Jay, and P. Efthimiou. 2017. Recommendations for optimizing methotrexate treatment for patients with rheumatoid arthritis. Open Access Rheumatology: Research and Reviews 9: 67-79.

26. Bykerk, V.P., A.J. Ostor, J. Alvaro-Gracia, K. Pavelka, J.A. Roman Ivorra, W. Graninger, et al. 2015. Comparison of tocilizumab as monotherapy or with add-on disease-modifying antirheumatic drugs in patients with rheumatoid arthritis and inadequate responses to previous treatments: An open-label study close to clinical practice. Clinical Rheumatology 34 (3): 563-571.

27. Lu, B., D.J. Antoine, K. Kwan, P. Lundback, H. Wahamaa, H. Schierbeck, et al. 2014. JAK/STAT1 signaling promotes HMGB1 hyperacetylation and nuclear translocation. Proc Natl Acad Sci U S A. 111 (8): 3068-3073.

Publisher's Note Springer Nature remains neutral with regard to jurisdictional claims in published maps and institutional affiliations. 